\title{
Vacío existencial y riesgo suicida en pacientes con trastorno de personalidad borderline
}

\author{
Álvaro Eduardo Valdivia Pareja
}

El presente estudio tiene como finalidad describir las características existentes en el vacio existencial y el riesgo suicida en pacientes con trastorno de personalidad borderline. Los instrumentos utilizados para tal propósito son la Prueba Propósito en la Vida (PIL), la Escala de Intencionalidad Suicida (SIS) y el Protocolo de Evaluación del Diagnóstico del Trastorno de Personalidad Borderline. La muestra, intencional (no azarosa), estuvo conformada por 42 participantes, 32 mujeres y 10 varones, que al momento de la evaluación asistían al servicio de Diagnóstico y Consejo Psicológico (consulta externa) de un hospital psiquiátrico nacional de Lima. La metodología utilizada fue de tipo descriptiva, con diseño descriptivo comparativo. Los resultados muestran que la prueba Propósito en la Vida (PIL) y la prueba de Intencionalidad Suicida (SIS) presentan confiabilidad y validez de constructo.

\section{Vacío existencial / riesgo suicida / personalidad borderline}

\section{Existencial emptiness and suicidal risk in borderline personality patients}

The purpose of this study is to describe the characteristics that borderline personality disorder patients show in existential emptiness and suicidal risk. The instruments used were the Purpose in Life Test (PIL), Suicide Intent Scale (SIS) and the Borderline Personality Disorder Diagnosis Protocole. The nonprobabilistic sample consisted of 42 participants, 32 females and 10 males, whom, at the time of the study, were assisting to the Psychology and Diagnosis Service of a National Psychiatric Hospital of Lima. The method was descriptive, with a comparative-descriptive design. The results show that the PIL and SIS scales prove to have adequate reliability and construct validity.

Existential emptiness / suicidal risk / borderline personality 
Existe en la actualidad una gran cantidad de personas que están en constante riesgo de cometer un acto suicida. Las estadísticas nos muestran que dicho fenómeno se encuentra asociado a diferentes problemas de salud, ocupando así el cuarto lugar como causa de muertes violentas en Lima. Constituye, además, el 8\% de estas (Instituto de Medicina Legal, 2001).

Se señala que por cada mujer que se suicida lo hacen dos varones. En un estudio realizado en el Instituto Nacional de Salud Mental Honorio Delgado- Hideyo Noguchi en 1998 (Revista de Psiquiatría y Salud Mental "Hermilio Valdizán”. Vol VI, 2, 2005) se considera que los motivos más frecuentes relacionados con el suicidio son: conflictos conyugales $(29,6 \%)$, conflictos familiares $(27,6 \%)$ y conflictos sentimentales $(22,1 \%)$.

Asimismo, dicha fuente señala que los diagnósticos clínicos más frecuentes asociados al suicidio son: trastorno depresivo mayor $(39,4 \%)$, trastorno reactivo con síntomas depresivos $(35,5 \%)$ y abuso de alcohol y sustancias $(35,5 \%)$. Se presentan pensamientos suicidas en el $30,3 \%$ de la población. La mayoría de suicidios ocurre en Lima (80\%).

De otro lado, según la Organización Mundial de la Salud (OMS), en el año 2001 se registraron un millón de suicidios, una cifra de muertes que se encuentra por encima de las causadas por homicidios (500 mil) y guerras (230 mil) (Cabrejo, 2004).

Es indispensable saber que todos los intentos o amenazas de suicidio se deben tomar con mucha seriedad (Morón, 1980; Fawcett \& Shaughnessy, 1991), por lo que es necesario identificar las características clínicas de estos sujetos con el fin de evitar el aumento de las conductas de autoeliminación.

En este sentido, se advierte que negarse a atender a un paciente potencialmente suicida puede ser éticamente inapropiado, además de ser clínicamente inadecuado. La predicción del suicidio es muy difícil, por no decir imposible. Algunos pacientes minimizan o niegan la presencia de ideación o riesgo suicida cuando recién empiezan un tratamiento. Otros pueden no tener dichas tendencias al comienzo, pero pueden desarrollarlas durante el proceso psicoterapéutico (Barnett \& Porter, 1998).

En este contexto, el concepto de vacío existencial propuesto por la Escuela Logoterapéutica de Viktor Frankl adquiere un valor resaltante; por ello, sus alcances se consideran oportunos y adecuados para encontrar un propósito de vida que brinde calma y serenidad, rechazando así la psicología de corte nihilista, que no contempla el ámbito espiritual.

El trastorno de personalidad borderline es un concepto que ha sido utilizado durante mucho tiempo, sin embar- 
go, la definición que se le daba no era siempre la adecuada (Kernberg, 1984), dificultándose así la descripción y entendimiento de sus características clínicas. Esto motivó que se realizaran numerosas reinterpretaciones y estudios sobre dicho desorden, las cuales originaron asimismo esta investigación.

Como se desprende de lo anterior, la complejidad del tema hace imprescindible describir las características de estos pacientes para evitar la tasa de suicidios efectivamente consumados, brindando mayor atención al aspecto preventivo.

\section{OBJETIVO GENERAL}

El objetivo general de este trabajo es el de identificar las características existentes en el vacío existencial y el riesgo suicida en pacientes con trastorno de personalidad borderline.

\section{Objetivos específicos}

- Establecer las características del vacío existencial en pacientes con trastorno de personalidad borderline.

- Establecer las características del riesgo suicida en pacientes con trastorno de personalidad borderline.

- Describir las diferencias con respecto al vacío existencial y el riesgo suicida considerando las siguientes variables: sexo, edad, intentos previos de suicidio y letalidad del método suicida.

\section{HIPÓTESIS DE TRABAJO}

$\mathrm{H}_{1}$ : Existen diferencias en las características suicidas entre los niveles de vacío existencial en los pacientes con trastorno de personalidad borderline.

$\mathrm{H}_{2}$ : Los varones tienden a presentar mayor vacío existencial que las mujeres.

$\mathrm{H}_{3}$ : Los varones tienden a desarrollar mayor riesgo suicida que las mujeres.

$\mathrm{H}_{4}$ : Los pacientes menores de 24 años tienen a presentar mayor vacío existencial.

$\mathrm{H}_{5}$ : Los pacientes menores de 24 años tienden a desarrollar mayor riesgo suicida.

$\mathrm{H}_{6}$ : Las personas que han realizado por lo menos un intento previo de suicidio tienden a presentar mayor vacío existencial.

$\mathrm{H}_{7}$ : Las personas que han realizado por lo menos un intento previo de suicidio tienden a presentar mayor riesgo suicida.

$\mathrm{H}_{8}$ : Los sujetos que han realizado por los menos un intento previo de suicidio con una modalidad más letal tienden a presentar mayor riesgo suicida.

$\mathrm{H}_{9}$ : Los sujetos que han realizado por lo menos un intento previo de suicidio con una modalidad más letal tienden a presentar mayor riesgo suicida.

\section{MÉTOdo}

- Tipo de investigación: Cuantitativa, de tipo descriptiva. (Danhke, en 
Hernández, Fernández y Baptista, 2005).

- Diseño metodológico de investigación: Descriptivo comparativo (Sánchez y Reyes, 2006).

- Participantes: Los participantes de la investigación fueron pacientes hombres y mujeres de distintas edades que se encontraban en proceso de evaluación psicológica ambulatoria en el Servicio de Diagnóstico de un hospital psiquiátrico nacional de Lima durante el período agosto 2005-agosto 2006.

En la tabla 1 se aprecia la muestra conformada por 42 participantes, de los cuales los varones constituyeron el $28,6 \%$ (12 participantes), mientras que las mujeres el $71,4 \%$ (30 participantes).

Tabla 1

Composición de la muestra por sexo

\begin{tabular}{ccc}
\hline Sexo & Frecuencia & Porcentaje \\
\hline Masculino & 12 & 28,6 \\
Femenino & 30 & 71,4 \\
Total & 42 & 100,0 \\
\hline
\end{tabular}

La tabla 2 presenta la división realizada por grupos de edades. Así, se observa que los mayores porcentajes se encuentran en el grupo que oscila entre 18 y 23 años $(57,1 \%)$ y 24 y 29 años $(21,4 \%)$, reservándose valores menores para los grupos restantes.
Tabla 2

Composición de la muestra por grupo de edad

\begin{tabular}{ccc}
\hline $\begin{array}{c}\text { Grupo de } \\
\text { edad }\end{array}$ & Frecuencia & Porcentaje \\
\hline $18-23$ & 24 & 57,1 \\
$24-29$ & 9 & 21,4 \\
$30-35$ & 3 & 7,1 \\
$36-41$ & 5 & 11,9 \\
$48-53$ & 1 & 2,4 \\
\hline Total & 42 & 100,0 \\
\hline
\end{tabular}

En la tabla 3 se aprecian los distintos niveles educativos, observándose que la mayor cantidad de casos se presentan en el nivel de secundaria completa $(47,6 \%)$ y secundaria incompleta (19\%).

Tabla 3

Composición de la muestra por nivel educativo

\begin{tabular}{|c|c|c|}
\hline $\begin{array}{c}\text { Nivel } \\
\text { educativo }\end{array}$ & Frecuencia & Porcentaje \\
\hline $\begin{array}{l}\text { Primaria } \\
\text { completa }\end{array}$ & 1 & 2,4 \\
\hline $\begin{array}{l}\text { Secundaria } \\
\text { incompleta }\end{array}$ & 8 & 19,0 \\
\hline $\begin{array}{l}\text { Secundaria } \\
\text { completa }\end{array}$ & 20 & 47,6 \\
\hline $\begin{array}{l}\text { Técnica } \\
\text { incompleta }\end{array}$ & 5 & 11,9 \\
\hline $\begin{array}{l}\text { Técnica } \\
\text { completa }\end{array}$ & 3 & 7,1 \\
\hline $\begin{array}{l}\text { Superior } \\
\text { incompleta }\end{array}$ & 2 & 4,8 \\
\hline $\begin{array}{l}\text { Superior } \\
\text { completa }\end{array}$ & 3 & 7,1 \\
\hline Total & 42 & 100,0 \\
\hline
\end{tabular}


La tabla 4 presenta los distintos métodos empleados por los participantes en el último intento de suicidio ejecutado antes de la evaluación. Se observa que los mayores porcentajes se encuentran reservados para las modalidades de cortes en las muñecas $(35,5 \%)$ e ingesta de pastillas $(33,3 \%)$. Puntajes medios se presentan en los métodos de lanzamiento al vacío $(9,5 \%)$ y herida de bala $(7,1 \%)$.

Tabla 4

Composición de la muestra por tipo de intento de suicidio

\begin{tabular}{llc}
\hline \multicolumn{1}{c}{$\begin{array}{c}\text { Tipo de intento } \\
\text { de suicidio }\end{array}$} & Frecuencia & Porcentaje \\
\hline $\begin{array}{l}\text { Ingesta de pastillas } \\
\text { Cortes en }\end{array}$ & 14 & 33,3 \\
$\begin{array}{l}\text { las muñecas } \\
\text { Lanzamiento } \\
\text { al vacío }\end{array}$ & 15 & 35,7 \\
$\begin{array}{l}\text { Herida de bala } \\
\text { Ingesta de veneno }\end{array}$ & 2 & 9,5 \\
$\begin{array}{l}\text { Asfixia por } \\
\text { estrangulamiento }\end{array}$ & 2 & 4,1 \\
$\begin{array}{l}\text { Asfixia por } \\
\text { sumersión }\end{array}$ & 1 & 4,8 \\
Inyección de lejía & 1 & 2,4 \\
\hline \multicolumn{1}{c}{ Total } & 42 & 100,0 \\
\hline
\end{tabular}

En la tabla 5 se muestra la cantidad de intentos previos de suicidio ejecutados por los participantes. Los puntajes son similares entre ellos, observándose $38,1 \%$ para tres o más intentos previos; $33,3 \%$ para uno o dos intentos previos y $28,6 \%$ para ningún intento previo de suicidio.

Tabla 5

Composición de la muestra por intentos previos de suicidio

\begin{tabular}{ccc}
\hline $\begin{array}{c}\text { Intentos previos } \\
\text { de suicidio }\end{array}$ & \multicolumn{2}{c}{ Frecuencia Porcentaje } \\
\hline Ninguno & 12 & 28,6 \\
1 ó 2 & 14 & 33,3 \\
3 ó más & 16 & 38,1 \\
\hline Total & 42 & 100,0 \\
\hline
\end{tabular}

\section{Instrumentos empleados}

1. Prueba Propósito en la Vida (PIL). Evalúa el grado en el que el sentido o propósito en la vida han sido encontrados, así como la presencia de vacío existencial. Es sumamente útil para fines de investigación.

El instrumento está conformado por tres secciones. La parte A consiste en una escala psicométrica tipo Likert de 20 ítems, que evocan respuestas sobre el grado en que un individuo experimenta "propósito en la vida". Estos 20 ítems fueron seleccionados por medio del análisis ítem-test, y un estudio de la validez de constructo y concurrente de la prueba. La parte B ofrece una serie de frases incompletas respecto de la búsqueda de metas y sentido en la vida. La parte $\mathrm{C}$ consta de una pequeña autobiografía sobre cómo se han alcanzado o no dichas metas. 
Para efectos de la investigación se utilizó únicamente la parte $\mathrm{A}$.

La confiabilidad del PIL fue estudiada por Crumbaugh y Maholik (1964) por medio del método de división por mitades, obteniéndose un coeficiente de correlación de Pearson de 0.81 (Producto-Momento) y un coeficiente de 0.90 corregido a través del método Spearman-Brown $(\mathrm{N}=225,105$ "normales" y 120 pacientes). La misma relación fue establecida por Crumbaugh en 1968, obteniendo un coeficiente de correlación de Pearson de 0.85 (Producto-Momento) y de 0.92 corregido por el método de Spearman-Brown ( $\mathrm{N}=120$, párrocos protestantes). De modo similar, Bartschi (1999) halló resultados análogos en la adaptación realizada en nuestro medio.

La validez se analizó de dos maneras:

- Correlación entre los puntajes del PIL y las clasificaciones sobre el sentido de la vida manifestados por los pacientes según su terapeuta (el terapeuta completó el PIL de acuerdo con la forma como consideró que el paciente lo hubiera respondido, basado en sus conocimientos previos del caso).

- Correlación entre los puntajes del PIL y las clasificaciones realizadas por ministros sobre el ni- vel de sentido en la vida exhibido por un grupo de párrocos.

La comparación entre los resultados globales de los grupos "normales" ( $\mathrm{M}=112.42, \mathrm{~N}=805$, D. E. $=14.07)$ y los grupos de pacientes $(\mathrm{M}=92.60, \mathrm{~N}=346$, D. E. $=$ 21.34) mostraron la existencia de diferencias significativas $(\mathrm{p}<.001)$, encontrándose los resultados en la dirección esperada, evidenciando así adecuada validez de constructo. Asimismo, Bartschi (1999) halló similares resultados en la adaptación que hizo a nuestro medio.

2. Escala de Intencionalidad Suicida. Se trata de una entrevista semiestructurada cuyo propósito es medir la seriedad de intentos suicidas en distintos pacientes (utilizado mayormente en personas que han cometido intentos previos de suicidio). El SIS consiste en 15 ítems que cuantifican el comportamiento verbal y no verbal de pacientes antes y durante el más reciente intento suicida. Cada ítem se puntúa en una escala que va del 0 al 2 , con un puntaje que varía de 0 a 30 puntos.

La primera parte del SIS (ítems del 1 al 8) evalúa las circunstancias objetivas del intento suicida, incluyendo ítems con respecto a la preparación y la manera de ejecución del intento, el ambiente y diversas pautas que pueda brindar el paciente y facilite el descubrimiento del 
intento. Esta parte de la escala puede ser completada incluso retrospectivamente, para pacientes que han cometido un acto suicida (por ejemplo, revisando reportes médicos e historias clínicas).

La segunda parte del SIS (ítems 9 al 15) evalúa la percepción del paciente con respecto a la letalidad del método, las expectativas sobre la posibilidad de rescate e intervención, el grado de premeditación y el propósito supuesto del intento.

La tercera parte del SIS (ítems 16 al 20) evalúa las circunstancias subjetivas del intento. Esta parte de la escala no se puntúa, razón por la cual no se considera de modo cuantitativo para fines de investigación.

La confiabilidad del instrumento se estimó mediante el coeficiente alpha de Cronbach. Se realizaron dos análisis de ítems debido a que en la primera evaluación los ítems 1, 4 y 8 presentaban valores muy bajos, y, por lo tanto, no superaban el criterio de 0,20 (Kline, 1983). El segundo análisis permitió apreciar que la eliminación de dichos ítems incrementó de manera sustancial la confiabilidad, por lo que se optó por trabajar solo con los ítems consistentes.

Los resultados del análisis del área 1 del SIS (Circunstancias objetivas) indican que los ítems fluctúan entre 0,52 (SIS 3) y 0,36 (SIS 5), los cuales superan ampliamente los criterios establecidos por Kline (1993). La confiabilidad total del área alcanzó un valor de 0,71 , concluyendo así que esta es confiable.

Los resultados del área 2 del SIS (Autoinforme) señalan que los ítems fluctúan entre 0,82 (SIS 10) y 0,23 (SIS 15), los cuales superan el criterio de 0,20 . Dado que la confiabilidad total del área alcanza un valor total de 0,83 , es posible concluir que posee adecuada confiabilidad.

Se estudió la validez de constructo a través del Análisis Factorial, cuyo resultado indicó que existía un solo factor entre las dos partes de la prueba que permitía explicar el $75,78 \%$ de la varianza explicada, por lo cual se puede concluir que la prueba presenta validez de constructo.

\section{Protocolo de Evaluación del Diagnóstico del Trastorno de PeRSONALIDAD BorderLINE}

Debido a la dificultad de establecer con claridad el diagnóstico pertinente para la muestra de la investigación, el evaluador consideró de remarcada importancia utilizar tres métodos para concluir que el diagnóstico es el adecuado.

- Información brindada por el profesional asignado al caso.

- Revisión de historias clínicas (en los casos que eran permitidos). 
- Utilización de un protocolo de evaluación del trastorno.

La creación del protocolo obedece al criterio y exigencias propias que el investigador consideró necesarias para la investigación, basándose en los criterios diagnósticos del DSM-IV-TR (2001), así como por la asesoría de tres psicólogos clínicos familiarizados con el tema en cuestión.

\section{Procedimiento de ReCOlección de DATOS:}

La primera fase de la evaluación tuvo como propósito primordial la verificación de las tres fuentes de información para el diagnóstico apropiado: la revisión del diagnóstico provisional brindado por el especialista, el chequeo de los criterios establecidos por medio del protocolo de evaluación y la revisión de las historias clínicas para elegir únicamente a los participantes que cumplieran con los requisitos establecidos para el estudio.

Concluida dicha verificación, se inició la recolección en sí misma, la cual, en todos los casos, se dio con la citación del o la participante a una hora establecida. Las evaluaciones se realizaron en una sola cita en un tiempo aproximado de 20 minutos.

La aplicación de los instrumentos se inició con la entrevista semiestructurada SIS, tomando en cuenta como mínimo un intento previo de suicidio. El evaluador entrevistó personalmente a los pacientes. La duración de esta fase fue de 10 minutos aproximadamente. Posteriormente se dio paso a la aplicación del PIL a cargo de cada uno de los participantes en un tiempo aproximado de 12 minutos.

\section{Resultados:}

La primera hipótesis planteada se contrasta con los resultados de la tabla 6 , que muestra el análisis comparativo del SIS según el nivel de vacío existencial. Se aprecia que existen diferencias estadísticas significativas en el SIS Total $(\mathrm{t}=-2.03 \mathrm{p}<.05)$, así como en el área de autoinforme $(\mathrm{t}=-3.04 \mathrm{p}<-05)$, las

Tabla 6

Análisis comparativos del SIS según niveles de vacío existencial

\begin{tabular}{lccccccc}
\hline \multirow{2}{*}{ Variables } & \multicolumn{2}{c}{$\begin{array}{c}\text { Vació existencial } \\
\text { profundo } \\
\mathrm{N}=34\end{array}$} & $\begin{array}{c}\text { Vacío existencial } \\
\text { moderado } \\
\mathrm{N}=7\end{array}$ & $\mathrm{t}$ & Sig. \\
\cline { 2 - 6 } & $\mathrm{M}$ & D.E. & $\mathrm{M}$ & $\mathrm{D} . \mathrm{E}$. & & \\
\hline Total SIS & 12.41 & 5.62 & 17.00 & 4.40 & -2.03 & 0.049 \\
Circunstancias objetivas (SIS 1) & 4.14 & 2.54 & 5.86 & 3.02 & -1.57 & 0.123 \\
Autoinforme (SIS 2) & 8.26 & 3.90 & 11.14 & 1.77 & -3.04 & 0.006 \\
\hline
\end{tabular}


cuales indican que el nivel de vacío existencial moderado presenta valores más elevados que el vacío existencial profundo, relacionándose así con un mayor riesgo suicida. En conclusión, la hipótesis 1 es aceptada.

Respecto de la segunda hipótesis, el resultado de la tabla 7 permite apreciar que no existen diferencias estadísticas significativas $(t=0.29 p>.05)$. En conclusión, la hipótesis 2 es rechazada.

La tercera hipótesis es contrastada con los resultados de la tabla 8 , que indican que solo existen diferencias esta- dísticas significativas en el caso del SIS total $(T=2.05 \mathrm{p}<.05)$, notándose que los hombres alcanzan una media aritmética mayor $(\mathrm{M}=15.83 \mathrm{D}$. E. $=$ 4.74) que las mujeres $(\mathrm{M}=12.07 \mathrm{D} . \mathrm{E}$. $=5.62$ ), lo que indica un mayor riesgo suicida. En conclusión, la hipótesis 3 es aceptada.

La cuarta hipótesis planteada en el estudio se contrasta con los resultados de la tabla 9, que muestran que no existen diferencias estadísticas significativas en el vacío existencial respecto a los grupos de edad $(\mathrm{t}=0.23 \mathrm{p}>.05)$. En conclusión, la hipótesis 4 es rechazada.

Tabla 7

Prueba $t$ de Student del vacío existencial según el sexo

\begin{tabular}{|c|c|c|c|c|c|c|}
\hline \multirow[t]{2}{*}{ Variable } & \multicolumn{2}{|c|}{$\begin{array}{l}\text { Masculino } \\
\mathrm{N}=12\end{array}$} & \multicolumn{2}{|c|}{$\begin{array}{c}\text { Femenino } \\
\mathrm{N}=30\end{array}$} & \multirow[t]{2}{*}{$\mathrm{t}$} & \multirow[t]{2}{*}{ Sig. } \\
\hline & $\mathrm{M}$ & D.E. & $\mathrm{M}$ & D.E. & & \\
\hline PIL & 68.25 & 19.86 & 70.17 & 19.47 & 0.29 & 0.776 \\
\hline
\end{tabular}

Tabla 8

Prueba t de Student del riesgo suicida según el sexo

\begin{tabular}{|c|c|c|c|c|c|c|}
\hline \multirow[t]{2}{*}{ Variable } & \multicolumn{2}{|c|}{$\begin{array}{l}\text { Masculino } \\
\mathrm{N}=12\end{array}$} & \multicolumn{2}{|c|}{$\begin{array}{c}\text { Femenino } \\
N=30\end{array}$} & \multirow[t]{2}{*}{$t$} & \multirow[t]{2}{*}{ Sig. } \\
\hline & M & D.E. & M & D.E. & & \\
\hline SIS 1 & 5.42 & 2.61 & 4.03 & 2.58 & 1.57 & 0.125 \\
\hline SIS 2 & 10.42 & 3.03 & 8.03 & 3.82 & 1.92 & 0.238 \\
\hline Total SIS & 15.83 & 4.74 & 12.07 & 5.62 & 2.05 & 0.048 \\
\hline
\end{tabular}

Tabla 9

Prueba $t$ de Student del vacío existencial según el grupo de edad

\begin{tabular}{|c|c|c|c|c|c|c|}
\hline \multirow[t]{2}{*}{ Variable } & \multicolumn{2}{|c|}{$\begin{array}{c}18-23 \text { años } \\
N=24\end{array}$} & \multicolumn{2}{|c|}{$\begin{array}{c}24 \text { años a más } \\
\quad \mathrm{N}=18\end{array}$} & \multirow[t]{2}{*}{$\mathrm{t}$} & \multirow[t]{2}{*}{ Sig. } \\
\hline & M & D.E. & M & D.E. & & \\
\hline PIL & 70,21 & 20,65 & 68,83 & 18,03 & 0.23 & 0.823 \\
\hline
\end{tabular}


Con respecto a la quinta hipótesis, los resultados de la tabla 10 señalan que no existen diferencias estadísticas significativas en caso alguno. En conclusión, la hipótesis 5 es rechazada.

La sexta hipótesis se contrasta con el resultado de la tabla 11, el cual indi- ca que no existen diferencias estadísticas significativas $(F=0.44 p>.05)$. En conclusión, la hipótesis 6 es rechazada.

Respecto de la séptima hipótesis, la tabla 12 presenta el análisis de varianza de un factor del riesgo suicida, que indica que no existen diferencias esta-

Tabla 10

Prueba $\mathbf{t}$ de Student del riesgo suicida según el grupo de edad

\begin{tabular}{|c|c|c|c|c|c|c|}
\hline \multirow[t]{2}{*}{ Variable } & \multicolumn{2}{|c|}{$\begin{array}{c}18-23 \text { años } \\
N=24\end{array}$} & \multicolumn{2}{|c|}{$\begin{array}{c}24 \text { años a más } \\
N=18\end{array}$} & \multirow[t]{2}{*}{$t$} & \multirow[t]{2}{*}{ Sig. } \\
\hline & M & D.E. & M & D.E. & & \\
\hline SIS 1 & 4,79 & 2,65 & 3,94 & 2,62 & 1.03 & 0.308 \\
\hline SIS2 & 8,83 & 3,07 & 8,55 & 4,57 & 0.24 & 0.815 \\
\hline Total SIS & 13,63 & 4,88 & 12,50 & 6,53 & 0.64 & 0.526 \\
\hline
\end{tabular}

Tabla 11

Análisis de varianza de un factor del vacío existencial según cantidad de intentos previos de suicidio

\begin{tabular}{|c|c|c|c|c|c|}
\hline \multirow{4}{*}{ Variable } & Ningún & 1 ó 2 & 3 ó más & \multirow{4}{*}{$\mathrm{F}$} & \multirow{4}{*}{ Sig. } \\
\hline & \multirow{2}{*}{\multicolumn{3}{|c|}{$\begin{array}{ccc}\text { intento previo intentos previos } & \text { intentos previos } \\
\mathrm{N}=12 & \mathrm{~N}=14 & \mathrm{~N}=16\end{array}$}} & & \\
\hline & & & & & \\
\hline & M & M. & M. & & \\
\hline PIL & 73.42 & 66.21 & 69.75 & 0.44 & 0.650 \\
\hline
\end{tabular}

Tabla 12

Análisis de varianza de un factor del riesgo suicida según cantidad de intentos previos de suicidio

\begin{tabular}{lccccc}
\hline Variable & $\begin{array}{c}\text { Ningún } \\
\text { intento previo } \\
\mathrm{N}=12\end{array}$ & $\begin{array}{c}\text { 1 ó } 2 \\
\text { intentos previos } \\
\mathrm{N}=14\end{array}$ & $\begin{array}{c}\text { 3 ó más } \\
\text { intentos previos } \\
\mathrm{N}=16\end{array}$ & $\mathrm{~F}$ & \multirow{2}{*}{ Sig. } \\
\cline { 2 - 4 } & $\mathrm{M}$ & $\mathrm{M}$ & $\mathrm{M}$. & & \\
\hline SIS 1 & 3.92 & 3.71 & 5.44 & 2.01 & 0.148 \\
SIS2 & $6.50 \mathrm{a}$ & $8.07 \mathrm{~b}$ & $10.93 \mathrm{ab}$ & 6.53 & 0.004 \\
Total SIS & $10.41 \mathrm{a}$ & $11.79 \mathrm{~b}$ & $16.38 \mathrm{ab}$ & 5.49 & 0.008 \\
\hline
\end{tabular}

Nota: Se utilizó como medida de contraste de hipótesis a posteriori el test de las mínimas diferencias (LSD) a nivel de significación del 0.05: a, b. 
dísticas significativas entre los grupos de acuerdo con la cantidad de intentos previos de suicidio en el caso del SIS 1 $(\mathrm{F}=2.01 \mathrm{p}>.05)$. Se encuentra, además, que existen diferencias estadísticas significativas en el caso del SIS 2 $(\mathrm{F}=6.53 \mathrm{p}<.001)$ y el SIS total $(\mathrm{F}=$ $5.49 \mathrm{p}<.001)$, notándose en ambos casos que el grupo de tres o más intentos previos de suicidio supera al grupo de uno o dos intentos, y el grupo de ningún intento previo. En conclusión, la hipótesis 7 es aceptada.

Para el contraste de la octava hipótesis se clasificaron los tipos de intentos de suicidio como de mayor letalidad a los métodos de cortes en las muñecas, arrojarse al vacío, herida de bala, ingesta de veneno e inyección de le- jía, mientras que en el grupo de menor letalidad fueron consideradas las modalidades de ingesta de pastillas, asfixia por estrangulamiento y asfixia por sumersión. Los resultados presentados en la tabla 13 indican que no existen diferencias estadísticas significativas en el vacío existencial de acuerdo con la letalidad del intento de suicidio $(\mathrm{t}=0.12 \mathrm{p}>.05)$. En conclusión, la hipótesis 8 es rechazada.

La novena hipótesis se contrasta con los resultados presentados en la tabla 14, que indican que no existen diferencias estadísticas significativas en el riesgo suicida en las comparaciones según el grado de letalidad del intento de suicidio. En conclusión, la hipótesis 9 es rechazada.

Tabla 13

Prueba t de Student del vacío existencial según la letalidad de los intentos de suicidio

\begin{tabular}{|c|c|c|c|c|c|c|}
\hline \multirow[t]{2}{*}{ Variable } & \multicolumn{2}{|c|}{$\begin{array}{l}\text { Menor letalidad } \\
\qquad \mathrm{N}=17\end{array}$} & \multicolumn{2}{|c|}{$\begin{array}{l}\text { Mayor letalidad } \\
\quad N=25\end{array}$} & \multirow[t]{2}{*}{$t$} & \multirow[t]{2}{*}{ Sig. } \\
\hline & M & D.E. & $M$ & D.E. & & \\
\hline PIL & 70.06 & 20.06 & 69.32 & 19.27 & 0.12 & 0.905 \\
\hline
\end{tabular}

Tabla 14

Prueba t de Student del riesgo suicida según la letalidad de los intentos de suicidio

\begin{tabular}{|c|c|c|c|c|c|c|}
\hline \multirow[t]{2}{*}{ Variable } & \multicolumn{2}{|c|}{$\begin{array}{c}\text { Menor letalidad } \\
\quad \mathrm{N}=17\end{array}$} & \multicolumn{2}{|c|}{$\begin{array}{l}\text { Mayor letalidad } \\
\quad N=25\end{array}$} & \multirow[t]{2}{*}{$\mathrm{t}$} & \multirow[t]{2}{*}{ Sig. } \\
\hline & M & D.E. & M & D.E. & & \\
\hline SIS 1 & 3.82 & 2.53 & 4.84 & 2.67 & -1.24 & 0.224 \\
\hline SIS2 & 8.00 & 4.34 & 9.20 & 3.27 & -1.02 & 0.313 \\
\hline Total SIS & 11.82 & 6.17 & 14.04 & 5.11 & -1.27 & 0.212 \\
\hline
\end{tabular}


Tabla 15

Análisis comparativo del PIL con los resultados hallados en la investigación de Bartschi (1999) en estudiantes universitarios

\begin{tabular}{lccccc}
\hline Variable & M. & D.E. & Valor teórico & t & Sig. \\
\hline PIL & 69.92 & 13.35 & 97.5 & -9.34 & 0.000 \\
\hline
\end{tabular}

\section{Análisis complementario}

En la tabla 15 se presenta la comparación entre los niveles del PIL de la presente investigación y la realizada por Bartschi (1999) con estudiantes universitarios, donde se encontró una media aritmética de 97.5 (que en el análisis constituye el valor teórico). El contraste indica que existen diferencias estadísticas significativas $(\mathrm{t}=-9.34 \mathrm{p}<$ .05), apreciándose que el nivel del PIL en la presente investigación es menor, lo que denota que los pacientes con trastorno de personalidad borderline presentan menor propósito en la vida, es decir, un mayor vacío existencial.

\section{DISCUSIÓN}

Se aprecia que la primera hipótesis es aceptada, observándose mayor riesgo suicida en los pacientes con vacío existencial moderado que en aquellos con vacío existencial profundo. Esto podría deberse al hecho de que dichos pacientes tienden a mostrar habitualmente presencia conductual precipitada, razón por la cual pueden desencadenar ciertos niveles de energía desordenada en explosiones repentinas, imprevistas e impulsivas (Millon, 2002), lo cual es consistente con los hallazgos de Yen (2004) acerca de las características diagnósticas asociadas al riesgo suicida en estos pacientes (inestabilidad emocional, alteración de la identidad e impulsividad). Así, se podría plantear que el nivel profundo de vacío existencial no se asocia de manera significativa con el riesgo suicida, ya que en este estado es probable que las características mencionadas (en particular, la impulsividad) se vean reducidas o suprimidas temporalmente, denotándose menor energía para realizar conductas repentinas de autoaniquilación. Sin embargo, el nivel de vacío existencial moderado permitiría que la impulsividad, al igual que otras características asociadas al riesgo suicida, afloren de manera repentina.

Es conocido, además, que el estado de ánimo depresivo moderado o severo (el cual podría relacionarse con el vacío existencial profundo) tiende a dificultar no solo el repertorio conductual habitual, sino que también influye en los procesos asociativos del pensamiento, es decir, en las cogniciones, disminuyendo las posibilidades de cometer un acto suicida en virtud de la 
falta de energía presente y de la dificultad para activar esquemas de pensamiento que comúnmente se presentan en estos pacientes y que se asocian al riesgo suicida (Freeman et al., 1990), como el esquema de "Maldad" planteado por Newman (1998) o los planteamientos de Beck, desde la perspectiva cognitiva (1975), o de Litman y Tabacknick (en Morón, 1980), desde la perspectiva psicoanalítica; sin embargo, esto no se puede afirmar de manera categórica, motivo por el cual un examen mental que explore ideación o riesgo suicida es siempre necesario para evitar que se cometa alguna acción de este tipo.

No obstante, es necesario recordar que no siempre los desórdenes afectivos o los estados de ánimo disfuncionales tienen una raíz psicológica, sino que, en muchos casos, es la dimensión espiritual la que está afectada, pero ya que esta no puede enfermarse, su conexión directa con las dimensiones física y psicológica del ser humano hace que sea justamente en ellas en las que se manifieste la patología por medio de conductas inadecuadas, como los intentos de suicidio (Frank1, 1990).

De esta manera, al explorar los niveles de vacío existencial, se podría sostener que su nivel profundo estaría más relacionado con un desorden de origen espiritual, motivo por el cual las conductas suicidas (realizadas en las dimensiones física y psicológica, respectivamente) tenderían a presentarse más en el nivel moderado. La razón de ello es que en este nivel la conexión del ámbito espiritual con las demás dimensiones es más estrecha y logra manifestarse de forma más habitual. Así, en el caso de presentarse vacío existencial profundo, la forma de trabajarlo es principalmente penetrando el ámbito espiritual por medio de la logoterapia como terapia específica de neurosis noógenas (Frankl, 1990), mientras que en el caso del vacío existencial moderado se podría empezar por los niveles físico y psicológico, sin olvidar, claro está, un análisis existencial posterior de corte netamente espiritual.

La segunda hipótesis es rechazada. Esto podría deberse a que la conexión tridimensional del ser humano planteada por Frankl (1990) es idéntica para hombres y para mujeres, ya que ambos son seres humanos y comparten las mismas aproximaciones a la dimensión espiritual, aun cuando pudiesen existir diferencias de género que se manifiesten en las dimensiones física y psicológica.

Con respecto al planteamiento de la tercera hipótesis, se concluye que esta es aceptada. Estos resultados son consistentes con lo hallado por el Instituto de Medicina Legal (2001), el cual señala que por cada mujer que se suicida se suicidan dos varones.

Estos hallazgos podrían tener distintas explicaciones. Para Clemente y González (1996), por ejemplo, la relación entre necesidades humanas y ries- 
go suicida es significativa. Dicha clasificación señala que la ausencia de satisfacción de determinadas necesidades (primarias, de apoyo social y desarrollo personal) puede influir en la decisión suicida del sujeto. Es importante señalar que en nuestro medio, la satisfacción de dichas necesidades, en particular las concernientes a vivienda, salud, alimentación, etcétera (primarias) y las de desarrollo personal (educación, trabajo, competencia, etcétera) son mayoritariamente realizadas por los varones o "jefes de la familia". De esta manera, la presión psicológica en estos casos es determinante en el nivel de estrés percibido. Así, al encontrar dificultades en el ajuste al estrés, además de las características de personalidad presentes, no es fácil hallar una solución pertinente, optando, en algunos casos, por cometer un acto suicida.

En este sentido, y debido al pensamiento patriarcal dominante en nuestro medio, se vislumbran dificultades en la satisfacción de necesidades de apoyo social en los varones, ya que dicho prejuicio hace que la idea de que "las mujeres buscan ayuda y los hombres no" se fortalezca, mermando los posibles canales de ayuda (particularmente en las clases socioeconómicas bajas).

El planteamiento de la cuarta hipótesis es rechazado. Esto podría explicarse debido a que la etapa de desarrollo en la cual estos sujetos se encuentran (finales de la adolescencia a comienzos de la juventud), los cambios en el aspecto físico, en las formas de pensar, en los sentimientos que emanan, así como en la búsqueda de identidad, sensaciones y exploración de diversas índoles (Papalia, 2001) se contrapone directamente al aburrimiento crónico planteado por Frankl (1990), el cual es un síntoma cardinal del vacío existencial.

La quinta hipótesis es rechazada. Ello podría explicarse tomando en cuenta un planteamiento similar al ofrecido en la cuarta hipótesis, con respecto a la consolidación de la personalidad y de las características que se asocian a esta.

La sexta hipótesis es rechazada. Se podría señalar que los hallazgos de Yen (2004) acerca de las características diagnósticas asociadas al riesgo suicida en estos pacientes son consistentes con este hecho, al no asociar el vacío con la conducta suicida. Se desconoce además si existe relación alguna entre dichas variables psicológicas en nuestro medio.

Con respecto al planteamiento de la séptima hipótesis, se aprecia que esta es aceptada. Este hallazgo es consistente con lo propuesto por Beck (1974), Morón (1980), Fawcett y Shaughnessy (1991) y Oldham (2006).

Asimismo, se puede señalar que el hecho de tener una historia de intentos previos de suicidio es significativo, ya que el sujeto, al haber realizado el acto una vez, encuentra mayor facilidad pa- 
ra ejecutarlo nuevamente, pues ya hizo el análisis respectivo que todo suicida realiza (Beck, 1975; Litman y Tabacknick en Morón, 1980; Morón, 1980), y que es, en algunos casos, el motivo por el cual puede desistir. Esto ocurre, sobre todo, cuando el suicidio era esperado, pero no consumado por factores ajenos al suicida. De ser así, la frustración proveniente de no haber podido realizar exitosamente el intento podría también aumentar las posibilidades de que se vuelva a realizar.

En este planteamiento, el vacío existencial podría jugar un rol muy importante, ya que, según lo planteado por Frankl (1990), el aburrimiento podría ser "mortal". El autor menciona que los suicidios podrían atribuirse, en última instancia, a aquel vacío interior que responde a la frustración existencial. Y sería justamente en este estado de aburrimiento constante de la vida en el cual el sujeto podría intentar repetidamente acabar con su vida.

Se debe recordar, además, que los hallazgos de Yen (2004) son muy importantes en este contexto, ya que al tratarse de sujetos con un trastorno de personalidad, los criterios diagnósticos que con él se asocian se mantienen persistentemente, como por ejemplo la impulsividad, alteración de la identidad e inestabilidad emocional, criterios que justamente se hallan asociados al riesgo suicida, según la autora.
Así, al presentarse continuamente estas características, la posibilidad de que se produzcan futuros intentos de suicidio es bastante más alta. Por esta razón, el proceso psicoterapéutico de pacientes con historia de intentos de suicidio debe centrarse primordialmente en dichos intentos de autoaniquilación, pero también en las características que se asocian a estos, a fin de disminuir el riesgo suicida.

La octava hipótesis es rechazada. Podría sustentarse que la letalidad del intento de suicidio no obedece necesariamente a una mayor frustración existencial, ya que el objetivo en todos los casos es la muerte (independientemente del nivel de vacío existencial que se pudiese presentar).

De esta manera, la conexión de la dimensión espiritual con la física y psicológica (Frankl, 1990) no intervendría con la elección de un método u otro. Se debe, además, tomar en cuenta el aspecto subjetivo del suicida, pues no para todos el término "letal" (referido al método empleado) significa lo mismo.

Es decir, la modalidad de ingesta de pastillas, por ejemplo, podría ser la menos letal, pero existen personas que la consideran más efectiva que la de cortes en las muñecas o ingesta de veneno, aun cuando se sabe que una muerte por ingesta de pastillas es altamente improbable. Así, este aspecto subjetivista de la letalidad del método impide que exista una real conexión con el vacío existencial. 
La novena hipótesis es rechazada. Los argumentos para esto son similares a los de la octava hipótesis; se alude a la subjetividad de la letalidad del método suicida y su escasa relación con futuros intentos debido a esto.

Por último, el análisis complementario evidencia que los participantes de la muestra de la presente investigación presentan un menor propósito en la vida, es decir, un mayor vacío existencial, al ser comparados con la muestra de Bartschi (1999) con estudiantes universitarios.

Este hallazgo se encuentra en la misma dirección que los datos extraídos del DSM-IV-TR (2001) con respecto a estos pacientes, e incluso cuando el criterio de "sentimientos crónicos de vacío" planteado en el Manual diagnóstico y estadístico de los trastornos mentales no es precisamente el mismo término acuñado por Frankl (1990) como "vacío existencial", comparten gran parte de este, evidenciándose la diferencia en el énfasis que pone Frankl con respecto a la dimensión espiritual del ser humano.

Trabajar con este tipo de pacientes es difícil, y debe ser asumido como un reto en el proceso de terapia. En este punto, es importante reconocer las emociones que emanan del terapeuta con respecto al paciente, ya que, en muchos casos, el desorden es tan disfuncional y existen tantos problemas asociados, que se puede pensar que "no tienen salvación" o que "su vida será siempre igual, hasta el día en que acabe por matarse de verdad", causando esto sentimientos de incompetencia, inutilidad e impotencia, que podrían interferir negativamente en el proceso.

Finalmente, es importante reconocer que una adecuada labor preventiva sería la clave para minimizar el riesgo suicida en cualquier población; sin embargo, y al tratarse de pacientes particularmente vulnerables, es necesario un trabajo sistémico e interdisciplinario, independiente del trabajo personal, que pueda afianzar las redes de soporte social con las que cuenta la persona, solo así se podría realmente reducir la tasa de acciones suicidas y conductas autoagresivas (ya que estas, si bien no han sido parte de la investigación, juegan un rol muy importante, pues tienden a presentarse de manera bastante recurrente en estos pacientes, $y$, en la gran mayoría de casos, el acto suicida puede no ser más que el paso final de un continuo autoagresivo que se inicia con dichas conductas de automutilación, incluso si se trata de comportamientos de manipulación o chantaje emocional) que se realizan en la actualidad.

\section{REFERENCIAS}

Asociación Psiquiátrica Americana (1994). Manual diagnóstico y estadístico de los trastornos mentales. 4. ${ }^{a}$ edición (DSM-IV). Washington. 
Asociación Psiquiátrica Americana (2000). Manual diagnóstico y estadístico de los trastornos mentales. 4. ${ }^{a}$ edición. Texto revisado (DSMIV-TR). Washington.

Asociación Psiquiátrica Americana (2001). Manual diagnóstico y estadístico de los trastornos mentales. 4. ${ }^{\text {a }}$ edición. Texto revisado (DSMIV-TR) Breviario. Criterios diagnósticos. Masson.

Barlow, D. \& Durand, M. (2003). Psicopatología. 3. ${ }^{\text {a }}$ edición. Madrid: Thomson.

Barnett, J. \& Porter, J. (1998). The suicidal patient: Clinical and risk management strategies. En: Vandecreek, L. Innovations in Clinical Practice: A Source Book. Vol. 16, pp. 95-107. Sarasota: Professional Resource Press.

Bartschi, C. (1999). Evaluación del propósito de vida y la búsqueda de metas noéticas desde el punto de vista de la logoterapia en estudiantes universitarios. Lima: Universidad de Lima.

Beck, A.; Beck, R. \& Kovacs, M. (1975). Classification of suicidal behaviours: Quantifying intent and medical lethality. American Journal of Psychiatry, 132 (3), 285-287.

Beck, R.; Morris, J. \& Beck, A. (1974). Cross-validation of the suicidal intent Scale. Psychological Reports, 34, 445-446.
Beck, A.; Schuyler, D. \& Herman I. (1974). Development of suicidal intent scales. En: Beck, A.; Resnik, H. \& Lettieri, D. (Eds.) The prediction of suicide. Bowie, MD: Charles Press.

Beck, A., Resnik, H. \& Lettieri, D. (1974). The prediction of suicide. Philadelphia: Charles Press.

Beck, A. (1979). Terapia cognitiva de la depresión. Madrid: Siglo XXI.

Belloch, A.; Sandín, B. \& Ramos, F. (1995). Manual de psicopatología. Vol. 2. Madrid: McGraw-Hill.

Cabrejo, J. (19.9.04). El suicidio: más fatal que la guerra. En: Diario $E l$ Comercio, El Dominical. Suplemento de Actualidad Cultural. Año 50, 287, p. 12.

Clemente, M. \& González, A. (1996). Suicidio: Una alternativa social. Madrid: Biblioteca Nueva.

Crumbaugh, J. (1968). Cross-validation of Purpose in Life Test based on Frankl's concepts. Journal of Individual Psychology. Vol. 24, pp. 74-81.

Crumbaugh, J. \& Maholick, L. (1964). An experimental study in existencialism: The psychometric approach to Frankl's concept of noogenic neurosis. Journal of Clinical Psychology. Vol. 20, 2, pp. 200-207. 
Crumbaugh, J. \& Maholick, L. (1969). Manual of instructions for the Purpose in Life Test. Murfreesboro: Psychometric Affiliates.

Durkheim, E. (1897). El suicidio. Madrid: Akal.

Fawcett, J. \& Shaughnessy, R. (1991). El paciente suicida. En: Flaherty, J.; Channon, R., \& Davis, J. Psiquiatría. Diagnóstico y tratamiento. Buenos Aires: Editorial Médica Panamericana, pp. 69-77.

Feldman, R. (1999). Psicología con aplicaciones a los países de habla hispana. 3. ${ }^{\mathrm{a}}$ edición. México: McGraw-Hill.

Frankl, V. (1970). Psicoanálisis y existencialismo. México: Fondo de Cultura Económica.

Frankl, V. (1987). Ante el vacío existencial: Hacia una humanización de la psicoterapia. Biblioteca de Psicología. Barcelona: Herder.

Frankl, V. (1990). Logoterapia y análisis existencial. Textos de cinco décadas. Barcelona: Herder.

Frankl, V. (1992). Teoría y terapia de las neurosis. Iniciación a la logoterapia y al análisis existencial. Barcelona: Herder.

Frankl, V. (1995). La presencia ignorada de Dios. Psicoterapia y religión. Barcelona: Herder.

Frankl, V. (1998). El hombre en busca de sentido. Barcelona: Herder.
Freeman, A.; Pretzer, J.; Fleming, B. \& Simon, K. (1990). Clinical applicactions of cognitive therapy. Nueva York-Londres: Plenum Press.

Guttmann, D. (1998). Logoterapia para profesionales. Trabajo social significativo. Bilbao: Desclée De Brouwer.

Hernández, R.; Fernández, C. \& Baptista, P. (2005). Metodología de la investigación. 3. ${ }^{\mathrm{a}}$ edición. México: McGraw-Hill.

Hospital Hermilio Valdizán (2005). Revista de Psiquiatría y Salud Mental “Hermilio Valdizán”. Vol. VI, 2, julio-diciembre.

Hospital Hermilio Valdizán (2006). 10 principales diagnósticos 2005 en consulta externa. Disponible: $<$ http://www.minsa.gob.pe/hhv/esta disticas.htm $>$.

Kernberg, O. (1984). Severe personality disorders. Psychotherapeutic strategies. Nueva York: Yale University Press.

Kline, P. (1993). The handbook of psychological testing. Londres: Routledge.

Kingberg, H. (2002). La llamada de la vida. La vida y la obra de Viktor Frankl. Barcelona: Integral.

Mann, J. John (2003). Neurobiology of suicidal behavior. Nature Reviews Neuroscience 4, 819-828, octubre 2003. 
Millon, T. (2002). Manual del Inventario Clínico Multiaxial de Million II. (3. ${ }^{\text {a }}$ edición). Publicaciones de Psicología Aplicada. Madrid: TEA Ediciones, S. A.

Millon, T. \& Everly, G. (1994). La personalidad y sus trastornos. Barcelona: Martínez Roca. Biblioteca de Psicología, Psiquiatría y Salud. Serie Clínica.

Morón, P. (1980). El suicidio. Serie: Colección de Psicología Preventiva. Buenos Aires: Ábaco.

Newman, C. (1998). Cognitive therapy for borderline personality disorder. En: Vandecreek, L. Innovations in clinical practice: A Source Book. Vol. 16, pp. 17-38. Sarasota: Professional Resource Press.

Oldham, M. John (2006). Borderline personality disorder and suicidality. American Journal of Psychiatry 163, 20-26, enero 2006.

Papalia, D.; Wendkos, S. \& Duskin, R. (2001). Psicología del desarrollo. 8. a edición. Bogotá: McGraw-Hill.

Ramos, F. \& González, H. (1993). Estrés, cognición y suicidio. En: Buendía, J. (Eds.). Estrés y psicopatología, pp. 113-130. Madrid: Pirámide, S.A.

Sánchez, H. \& Reyes, C. (2006). Metodología y diseños en la investigación científica. Lima: Visión Universitaria.
Sarró, B. \& De la Cruz, C. (1991). Los suicidios. Barcelona: Martínez Roca.

Sperry, L. (2003). Handbook of diagnosis and treatment of DSM-IV-TR personality disorders. 2. a edición. Nueva York: Brunner/Routledge.

Soloff, P.; Lynch, K.; Kelly, T.; Malone, K. \& Mann, J. (2000). Characteristics of suicide attempts of patients with major depressive episode and borderline personality disorder: A comparative study. American Journal of Psychiatry, 157, 601608, abril.

Tozzini, C. (1969). El suicidio. Buenos Aires: Depalma.

Yalom, I. (1984). Psicoterapia existencial. Barcelona: Herder.

Yen, Shirley; Shea, T.; Sanislow, C.; Grito, C.; Skodol, A.; Gunderson, J.; McGlashan, T.; Zanarini, M. \& Morey, L. (2004). Borderline personality disorder criteria associated with prospectively observed suicidal behaviour. American Journal of Psychiatry, 161, 1296-1298, julio. 